\title{
Management of severe ischemic cardiomyopathy: Left ventricular assist device as destination therapy versus conventional bypass and mitral valve surgery
}

\author{
Simon Maltais, MD, PhD, ${ }^{a}$ Vahtang Tchantchaleishvili, MD, ${ }^{\mathrm{b}}$ Hartzell V. Schaff, MD, ${ }^{\mathrm{c}}$ \\ Richard C. Daly, MD, ${ }^{c}$ Rakesh M. Suri, MD, PhD,${ }^{c}$ Joseph A. Dearani, MD, ${ }^{c}$ Yan Topilsky, MD, ${ }^{d}$ \\ John M. Stulak, MD, ${ }^{c}$ Lyle D. Joyce, MD, PhD, ${ }^{c}$ and Soon J. Park, MD, MSc ${ }^{c}$
}

\begin{abstract}
Objectives: Patients with severe ischemic cardiomyopathy (left ventricular ejection fraction $<25 \%$ ) and severe ischemic mitral regurgitation have a poor survival with medical therapy alone. Left ventricular assist device as destination therapy is reserved for patients who are too high risk for conventional surgery. We evaluated our outcomes with conventional surgery within this population and the comparative effectiveness of these 2 therapies.

Methods: We identified patients who underwent conventional surgery or left ventricular assist device as destination therapy for severe ischemic cardiomyopathy (left ventricular ejection fraction $<25 \%$ ) and severe mitral regurgitation. The era for conventional surgery spanned from 1993 to 2009 and from 2007 to 2011 for left ventricular assist device as destination therapy. We compared baseline patient characteristics and outcomes in terms of end-organ function and survival.
\end{abstract}

Results: A total of 88 patients were identified; 55 patients underwent conventional surgery (63\%), and 33 patients $(37 \%)$ received a left ventricular assist device as destination therapy. Patients who received left ventricular assist device as destination therapy had the increased prevalence of renal failure, inotrope dependency, and intra-aortic balloon support. Patients undergoing conventional surgery required longer ventilatory support, and patients receiving a left ventricular assist device required more reoperation for bleeding. Mortality rates were similar between the 2 groups at 30 days ( $7 \%$ in the conventional surgery group vs $3 \%$ in the left ventricular assist device as destination therapy group, $P=.65)$ and at 1 year $(22 \%$ in the conventional surgery group vs $15 \%$ in the left ventricular assist device as destination therapy group, $P=.58$ ). There was a trend toward improved survival in patients receiving a left ventricular assist device compared with the propensity-matched groups at 1 year $(94 \%$ vs $71 \%, P=.171)$.

Conclusions: The operative mortality and early survival after conventional surgery seem to be acceptable. For inoperable or prohibitive-risk patients, left ventricular assist device as destination therapy can be offered with similar outcomes. (J Thorac Cardiovasc Surg 2014;147:1246-50)

The presence of mitral regurgitation (MR) in the setting of left ventricular (LV) dysfunction is associated with increased mortality. ${ }^{1,2}$ With improvements in surgical techniques, mitral valve (MV) surgery for severe MR in the setting of advanced LV dysfunction has become an accepted option. ${ }^{3,4}$ However, in patients with severe ischemic cardiomyopathy, the surgical treatment of severe MR is still associated with increased operative risks and perioperative morbidity. ${ }^{5}$

\footnotetext{
From the Division of Cardiovascular Surgery, ${ }^{\text {a }}$ Vanderbilt University Medical Center, Nashville, Tenn; Division of Cardiac Surgery, ${ }^{\mathrm{b}}$ University of Rochester Medical Center, Rochester, NY; Division of Cardiovascular Surgery, ${ }^{\mathrm{c}}$ Mayo Clinic College of Medicine, Rochester, Minn; and Division of Cardiology, ${ }^{\mathrm{d}}$ Tel Aviv Sourasky Medical Center, Tel Aviv, Israel.

Disclosures: Dr Park received consulting fees from Thoratec. All other authors have nothing to disclose with regard to commercial support.

Received for publication Dec 20, 2012; revisions received March 25, 2013; accepted for publication April 9, 2013; available ahead of print June 13, 2013.

Address for reprints: Soon J. Park, MD, MSc, Division of Cardiovascular Surgery, Mayo Clinic College of Medicine, 200 First St SW, Rochester, MN 55905 (E-mail: Park.Soon@mayo.edu).

$0022-5223 / \$ 36.00$

Copyright (c) 2014 by The American Association for Thoracic Surgery http://dx.doi.org/10.1016/j.jtcvs.2013.04.012
}

Although studies have suggested a potential survival advantage of MV repair over replacement, ongoing debates remain regarding the effectiveness of mitral surgery in patients with end-stage ischemic cardiomyopathy ${ }^{6}$ and factors that really influence survival. ${ }^{7}$ Nonetheless, the management of patients with severe ischemic mitral regurgitation (IMR) and severe ischemic cardiomyopathy remains challenging and is still associated with poor outcomes. $^{8}$

Left ventricular assist devices (LVADs) have become the standard of care for patients with life-threatening heart failure refractory to optimal medical therapy. ${ }^{9}$ The HeartMate II LVAD (Thoratec, Pleasanton, Calif) has been approved for use as destination (permanent) therapy (DT) for patients with end-stage congestive heart failure who are ineligible for heart transplantation because of age, additional health problems, or other complications. ${ }^{10}$ Thus, the use of mechanical support devices in adult patients has recently become commonplace in many centers, and excellent reported outcomes have allowed the widespread use of the 

Abbreviations and Acronyms
$\mathrm{CABG}=$ coronary artery bypass grafting
$\mathrm{CS}=$ conventional surgery
DT $=$ destination therapy
IABP $=$ intra-aortic balloon pump
IMR = ischemic mitral regurgitation
$\mathrm{LV}=$ left ventricular
LVAD $=$ left ventricular assist device
LVEF $=$ left ventricular ejection fraction
MR = mitral regurgitation
MV = mitral valve

HeartMate II device in high-risk patients with end-stage cardiomyopathy. ${ }^{10}$

We conducted this study to understand the surgical results in patients with severe ischemic cardiomyopathy and severe IMR. The primary objective of this study was to evaluate surgical results in patients with severe ischemic LV dysfunction (left ventricular ejection fraction [LVEF] $\leq 25 \%$ ) and severe IMR. The secondary objective was to compare the effectiveness of conventional surgery (CS) (coronary artery bypass grafting [CABG] + MV surgery) versus LVAD as DT in this high-risk population.

\section{PATIENTS AND METHODS \\ Study Design}

This is a retrospective analysis of prospectively gathered data over more than a 10-year period (median, 3.6 years; range, 0-15 years). The Mayo Foundation Institutional Review Board approved this study, and individual consent was obtained for all patients included in this study. For patients in the conventional CABG + MV surgery group, the cardiac surgery database (1993 to 2009) at Mayo Clinic (Rochester, Minn) was used to identify a homogeneous study cohort of patients who underwent cardiac surgery for ischemic heart disease with severe IMR. We identified patients who underwent a combined CABG and MV repair or MV replacement first. Then, we excluded those who have had any one of the following conditions: LVEF greater than $25 \%$, concomitant mitral prolapse, infective endocarditis, congenital valvular heart disease, rheumatic valvular disease, or any degree of mitral stenosis. We used the same cardiac surgery database (2007-2011) to identify patients with end-stage ischemic cardiomyopathy with severe IMR who underwent implantation of a HeartMate II LVAD as DT during this period. Since the inception of our multidisciplinary LVAD program in 2007, LVAD DT was offered only to those who were deemed inappropriate for CS on the basis of having very poor to no coronary target vessels to improve myocardial ischemia. The frequency of CS per year was examined over time (pre- and post-LVAD DT). This study included only patients with the HeartMate II continuous-flow device (Thoratec, Pleasanton, Calif). All other device types were excluded from this study. All patients considered for LVAD DT were considered initially for conventional therapy. Myocardial viability in relation to suitable coronary targets and probability of mitral surgery were generally assessed first, and the decision was left to the surgeon's discretion to refer for advanced heart failure therapies.

\section{Definitions}

The cause of MR was ischemic; all operative and echocardiographic findings were reviewed in detail for all patients included in this study.
All patients were deemed to have severe IMR on the basis of leaflet tethering and prior myocardial infarction. Our institutional policy has been to preserve the posterior leaflet whenever possible with an increasing recent tendency toward preserving the anterior leaflet and by transposing it to the posterior annulus at the time of MV replacement. The operative mortality was defined as death from any cause within 30 days of surgery or during the same hospitalization. All patients in the MV repair group had the implantation of an undersized ring/band when applicable. In patients with mitral repairs, the ring was chosen according to the undersized intercommissural distance. In patients with posterior bands, the band was cut and undersized to the appropriate length. Prolonged intubation was defined as the need for mechanical ventilation greater than 48 hours. Of note, extubation protocol was not standardized among groups and left to physician preferences. Reported postoperative infections include superficial sternal, urinary tract, and pulmonary infections. Preoperative renal failure was defined as an uncorrected creatinine clearance less than $60 \mathrm{~mL} / \mathrm{min}$.

\section{Follow-up}

Patients were followed systematically using mailed questionnaires, telephone interview, or examination at the Mayo Clinic. Clinical follow-up was $100 \%$ complete. Mean follow-up among survivors was 4.2 years (range, 0-15.7 years).

\section{Statistical Analysis}

Mann-Whitney Wilcoxon rank-sum test was used to compare variables between patients with CS and patients with an LVAD. Categoric variables between 2 groups were compared using the Fisher exact test. The KaplanMeier method was used to construct survival curves, and survival was analyzed for both unmatched and matched populations. Differences in survival between 2 groups were obtained using the log-rank test. Statistical analysis was performed using R: A language and environment for statistical computing (R Foundation for Statistical Computing, Vienna, Austria).

\section{RESULTS}

\section{Patient Characteristics}

In this study, 88 patients were identified and had concomitant severe ischemic cardiomyopathy and severe IMR. From 1993 to 2009, 55 patients were labeled to have CS, because they had a combined CABG and MV (replacement or repair) procedure. From 2007 to 2011, 33 patients had an LVAD implanted as DT. The baseline characteristics of all patients and 2 subgroups according to the type of procedure (CS vs LVAD DT) are shown in Table 1. The Society of Thoracic Surgeons predicted mortality for patients in the CS group was $10.4 \%$. As shown in Table 1, age, preoperative LVEF, incidence of diabetes, and rate of redo surgeries were comparable between groups $(P>.05)$. However, as expected, there were significant observed differences between groups. Compared with patients with CS, patients in the LVAD DT group had a higher incidence of preoperative renal failure $(70 \%$ vs $15 \%, P<.001)$, a higher rate of preoperative intra-aortic balloon pump (IABP) (79\% vs $13 \%$, $P<.001)$, and a higher rate of preoperative inotrope dependency $(58 \%$ vs $15 \%, P<.001)$. Preoperative IABP is frequently used to optimize hemodynamic, right ventricular function, or kidney function before definitive LVAD therapy. We believe a short bridging ( $\leq 48$ hours) strategy with IABP helps optimize the preoperative condition before 
TABLE 1. Patient characteristics

\begin{tabular}{lccc}
\hline & $\begin{array}{c}\text { CS } \\
\mathbf{n}=\mathbf{5 5}(\%)\end{array}$ & $\begin{array}{c}\text { LVAD as DT } \\
\mathbf{n}=\mathbf{3 3}(\%)\end{array}$ & $\begin{array}{c}\boldsymbol{P} \\
\text { value }\end{array}$ \\
\hline Age (y)* & $70(67-75)$ & $71(67-73)$ & .68 \\
Gender (female) & $13(24)$ & $5(15)$ & .42 \\
LVEF (\%)* & $20(16-23)$ & $20(15-25)$ & .47 \\
Diabetes & $19(35)$ & $6(18)$ & .14 \\
Preoperative renal failure & $8(15)$ & $23(70)$ & $<.001$ \\
Preoperative inotropes & $8(15)$ & $19(58)$ & $<.001$ \\
Preoperative IABP & $7(13)$ & $26(79)$ & $<.001$ \\
Redo sternotomy & $11(20)$ & $8(24)$ & .79 \\
INTERMACS I or II & $\mathrm{n} / \mathrm{a}$ & $4(12)$ & $\mathrm{n} / \mathrm{a}$ \\
Lietz-Miller score (mean \pm SD) & $\mathrm{n} / \mathrm{a}$ & $9.3 \pm 0.7$ & $\mathrm{n} / \mathrm{a}$ \\
Kormos score (mean \pm SD) & $\mathrm{n} / \mathrm{a}$ & $1.7 \pm 0.2$ & $\mathrm{n} / \mathrm{a}$ \\
Matthews score (mean \pm SD) & $\mathrm{n} / \mathrm{a}$ & $1.0 \pm 0.2$ & $\mathrm{n} / \mathrm{a}$ \\
\hline
\end{tabular}

$C S$, Conventional surgery; $D T$, destination therapy; $I A B P$, intra-aortic balloon pump; INTERMACS, Interagency Registry for Mechanically Assisted Circulatory Support; $L V A D$, left ventricular assist device; $L V E F$, left ventricular ejection fraction; $n / a$, not available; $S D$, standard deviation. *Age and LVEF are presented using median with interquartile range for better representation.

LVAD implantation. In this study, a minority of patients $(12 \%, \mathrm{n}=4)$ were in cardiogenic shock before LVAD implantation.

\section{Operative Details}

In the CS group, 44 patients had MV repair (80\%) and 11 patients $(20 \%)$ had MV replacement. A total of 34 patients $(77 \%)$ in the MV repair group had the implantation of an undersized ring, and 10 patients $(23 \%)$ received an undersized band. In the replacement group, 7 patients $(64 \%)$ received a mechanical valve, and 4 patients $(36 \%)$ had a bioprosthesis. The mean number of anastomoses in the CS group was $2.7 \pm 1.3$. In the LVAD DT group, 33 patients had a HeartMate II continuous-flow device implanted.

\section{Early Comparative Outcomes}

We observed significant differences in outcomes comparing both populations (Table 2). Patients in the LVAD DT groups were more likely to undergo reoperation for bleeding $(64 \%$ vs $4 \%, P<.001)$. Patients in the CS group were more likely to have a prolonged intubation $(49 \%$ vs $12 \%, P<.001)$. The median length of stay was higher in the LVAD DT group (18 vs 10 days, $P<.001$ ), whereas the rate of postoperative infection and stroke was similar between groups $(P=.07$ and $P=.36$, respectively). Inhospital (or 30-day) and 1-year mortality were comparable between groups. Comparative survival using Kaplan-Meier analysis revealed no differences in estimated survival between groups (Figure 1, $P=.742$ ). Because of significant differences in baseline characteristics between groups, we compared 2 propensity-matched subgroups, controlled for preoperative differences of renal failure, IABP, and preoperative inotropes. Seventeen patients were identified in each subgroup. Although the difference in survival did not reach
TABLE 2. Postoperative outcome comparison

\begin{tabular}{lccc}
\hline & \multicolumn{1}{c}{ CS } & LVAD as DT & $\boldsymbol{P}$ \\
& $\mathbf{n = 5 5}$ & $\mathbf{n}=\mathbf{3 3}$ & value \\
\hline Reoperation bleeding & $2(4)$ & $21(64)$ & $<.001$ \\
Prolonged intubation & $27(49)$ & $4(12)$ & $<.001$ \\
Infection & $5(9)$ & $8(24)$ & .07 \\
Stroke & $2(4)$ & $3(9)$ & .36 \\
LOS (d)* & $10(7-16)$ & $18(13-22)$ & $<.001$ \\
Mortality (30 d) & $4(7)$ & $1(3)$ & .65 \\
Mortality (1 y) & $12(22)$ & $5(15)$ & .58 \\
Follow-up (d)* & $931(174-1600)$ & $341(209-566)$ & .08 \\
\hline$C S$, Conventional surgery; $D T$, destination therapy; LOS, length of stay; LVAD, left \\
ventricular assist device. *Continuous variables are presented using median with in- \\
terquartile range.
\end{tabular}

a statistical significance (Figure 2, $P=.171$ ), we observed a survival trend favoring the LVAD DT subgroup.

\section{DISCUSSION}

There have been several conflicting results in regard to the benefits of an MV intervention in patients with extremely low ejection fraction and ischemic cardiomyopathy. ${ }^{11}$ In this high-risk population, mitral surgery for severe MR during bypass surgery has become the accepted strategy for definitive management of patients with severe ischemic cardiomyopathy and severe IMR. Fedoruk and colleagues ${ }^{12}$ reported an observed $8.2 \%$ operative mortality with coronary bypass surgery and MV replacement/repair in patients with extremely low LVEF. Previously reported results by our group further suggest an acceptable 84\% 1-year and 51\% 5 -year survival in 179 patients with severe ischemic cardiomyopathy and severe MR. ${ }^{13}$ However, reported in-hospital mortality in this high-risk population has been reported to be as high as $27 \% .{ }^{14}$ In addition, several groups have observed only a modest improvement in LVEF and New York Heart Association functional class after surgery. ${ }^{14,15}$ Dahlberg and colleagues ${ }^{16}$ further observed that a low LVEF was an independent risk factor for mortality after concomitant CABG and MV surgery. Finally, despite effective repair using undersized mitral ring annuloplasty, several groups have reported early recurrence of MR in patients with LV dysfunction, thus reflecting the complexity of this disease and its treatment. ${ }^{17-19}$ We conducted this study to primarily understand our surgical outcomes in patients with severe ischemic cardiomyopathy and severe IMR. Our observed in-hospital or 30-day mortality in patients with conventional bypass and mitral surgery was $7 \%$, and the 1 -year mortality was $22 \%$. These results compare favorably to other reported series and reaffirm our ability to successfully treat these challenging patients with $\mathrm{CS}^{20,21}$

\section{Implications for Left Ventricular Assist Device as Destination Therapy}

LVADs have become the standard of care for patients with life-threatening heart failure refractory to optimal 


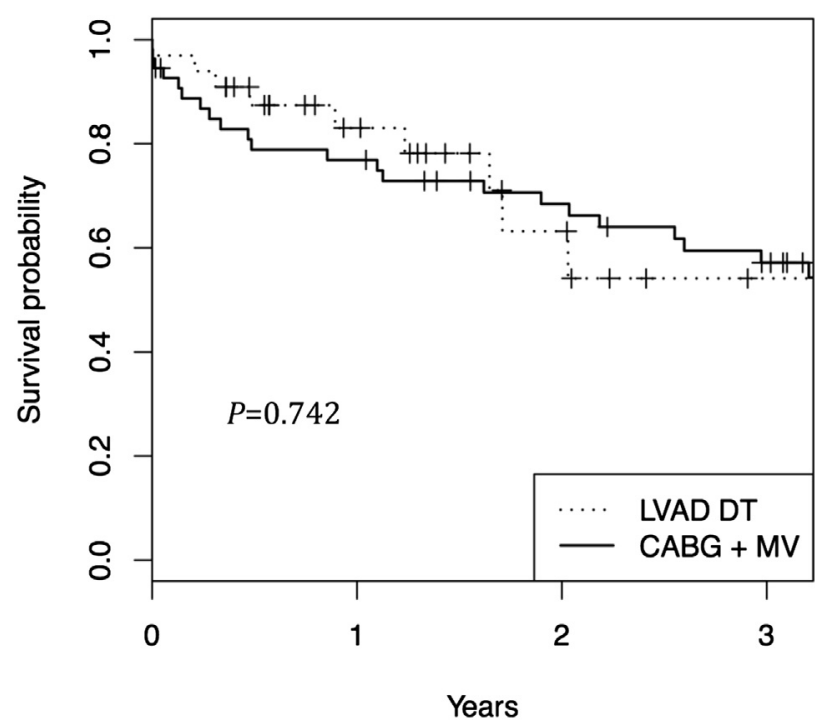

FIGURE 1. Overall survival. Comparative survival using Kaplan-Meier analysis revealing no differences in estimated survival between groups $(P=.742) . C A B G+M V$, Coronary artery bypass grafting + mitral valve; $L V A D D T$, left ventricular assist device destination therapy.

medical therapy. ${ }^{9}$ Within the last few years, there has been a paradigm shift in the use of long-term mechanical support devices in adult patients with advanced heart failure and end-stage cardiomyopathy. ${ }^{10}$ Several patients with ischemic myocardial dysfunction and severe ischemic MR are referred for long-term LVAD, primarily because of poor coronary targets or nonviability of the myocardium by noninvasive testing. It is thus reasonable to assess the implication of both surgical strategies in this high-risk population. To assess the impact of both therapies, we retrospectively compared the effectiveness of CS (CABG + MV surgery) with LVAD as destination. The patients in the LVAD DT group, in addition to having no coronary target vessels, were sicker (Table 1). Despite these differences, survival was comparable between the CS and LVAD DT groups at 30 days and 1 year $(7 \%$ vs $3 \% ; 22 \%$ vs $15 \% ; P=.65$ and $P=.58$ ) (Table 2). Within the propensity-matched population, a trend of improved survival was noted for the LVAD DT group (Figure 2). Given the small number of patients, it is not surprising that a statistically significant difference could not be demonstrated. It is clearly hypothesis generating in that LVAD DT might be preferable over CS in some appropriately selected subgroup of patients with advanced ischemic cardiomyopathy. Nevertheless, in the majority of patients with ischemic cardiomyopathy, CS composed of coronary bypass and MV repair/replacement seems to yield outcome comparable to that of LVAD DT. It now seems these 2 surgical options (CS and LVAD DT) need to be available as complementary therapy as we care for the entire spectrum of this challenging group of patients with advanced ischemic cardiomyopathy.

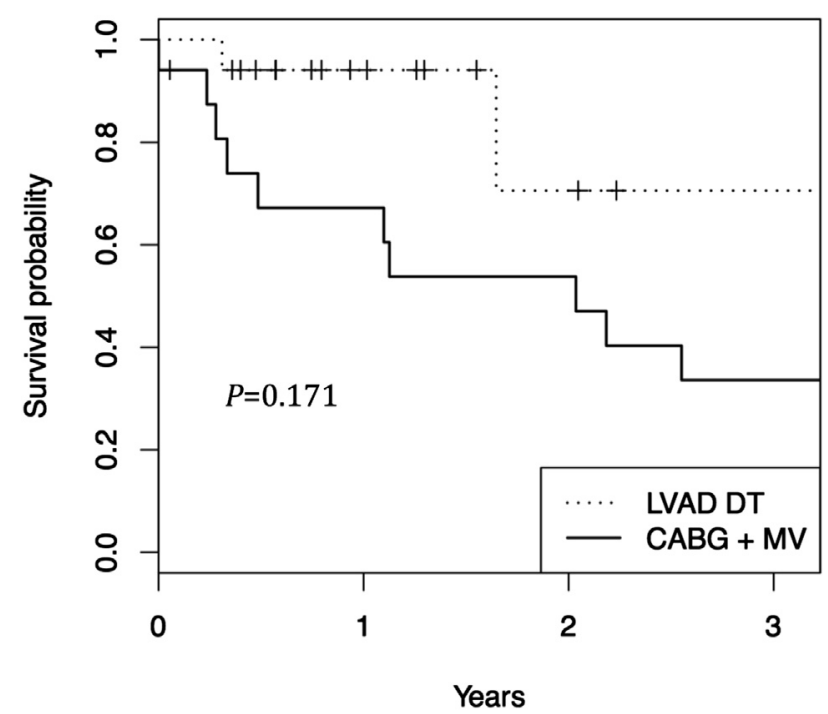

FIGURE 2. Survival in propensity-matched populations. After controlling for significant preoperative differences between groups, propensity-matched survival analysis did not reach statistical significance $(P=.171) . C A B G+$ $M V$, Coronary artery bypass grafting + mitral valve; $L V A D D T$, left ventricular assist device destination therapy.

\section{Study Limitations}

We recognize the retrospective nature of the study. The 2 groups underwent surgery in 2 different time periods without significant overlap, which makes the study more prone to systemic bias. This patient population represents different groups. A better understanding of this patient population will improve our decision-making process for these challenging patients. Survival comparisons need to be balanced with inherent selection differences between groups. Appropriate comparisons between these populations is challenging because conventional preoperative scoring systems are based on historical cohorts and were designed to address the specifics of each population without bridging the gap between surgical strategies. We attempted to correct for these differences by comparing 2 propensity-matched subgroups controlled for preoperative differences of renal failure, IABP, and preoperative inotropes. There is a need to develop a standardized preoperative scoring tool to better stratify patients and evaluate the impact of these 2 therapies. The median follow-up time also was shorter for patients receiving an LVAD (341 days) when compared with the CABG + MV surgery group (931 days). With sufficient statistical power and long enough follow-up time, the difference in survival could become significant.

\section{CONCLUSIONS}

We evaluated surgical results in patients with severe ischemic LV dysfunction and severe IMR, and compared the effectiveness of CS and LVAD as destination. The 
operative mortality and early survival after CS seem to be acceptable. For those who are inoperable or have prohibitive risks for surgery, LVAD DT can be offered with similar outcomes. Both of these modalities should be considered as we evaluate these challenging patients.

\section{References}

1. Robbins JD, Maniar PB, Cotts W, Parker MA, Bonow RO, Gheorghiade M Prevalence and severity of mitral regurgitation in chronic systolic heart failure. Am J Cardiol. 2003;91:360-2.

2. Trichon BH, Felker GM, Shaw LK, Cabell CH, O'Connor CM. Relation of frequency and severity of mitral regurgitation to survival among patients with LV systolic dysfunction and heart failure. Am J Cardiol. 2003;91: 538-43.

3. Oe M, Asou T, Kawachi Y, Kishizaki K, Fukamachi K, Sunagawa K, et al. Effects of preserving mitral apparatus on ventricular systolic function in mitral valve operations in dogs. J Thorac Cardiovasc Surg. 1993;106:1138-46.

4. David TE, Uden DE, Strauss HD. The importance of the mitral apparatus in left ventricular function after correction of mitral regurgitation. Circulation. 1983; 68:II76-82.

5. Koelling TM, Aaronson KD, Cody RJ, Bach DS, Armstrong WF. Prognostic significance of mitral regurgitation and tricuspid regurgitation in patients with left ventricular systolic dysfunction. Am Heart J. 2002;144:524-9.

6. Vassileva CM, Boley T, Markwell S, Hazelrigg S. Meta-analysis of short-term and long-term survival following repair versus replacement for ischemic mitral regurgitation. Eur J Cardiothorac Surg. 2011;39:295-303.

7. Maltais S, Schaff HV, Daly RC, Suri RM, Dearani JA, Sundt TM III, et al. Mitral regurgitation surgery in patients with ischemic cardiomyopathy and ischemic mitral regurgitation: factors that influence survival. $J$ Thorac Cardiovasc Surg. 2011;142:995-1001.

8. Rothenburger M, Rukosujew A, Hammel D, Dorenkamp A, Schmidt C, Schmid C, et al. Mitral valve surgery in patients with poor left ventricular function. Thorac Cardiovasc Surg. 2002;50:351-4.

9. Rose EA, Gelijns AC, Moskowitz AJ, Heitjan DF, Stevenson LW, Dembitsky W, et al. Long-term use of a left ventricular assist device for end-stage heart failure. N Engl J Med. 2001;345:1435-43.
10. Slaughter MS, Rogers JG, Milano CA, Russell SD, Conte JV, Feldman D, et al. Advanced heart failure treated with continuous-flow left ventricular assist device. N Engl J Med. 2009;361:2241-51.

11. Bouma W, van der Horst IC, Wijdh-den Hamer IJ, Erasmus ME, Zijlstra F, Mariani MA, et al. Chronic ischaemic mitral regurgitation. Current treatment results and new mechanism-based surgical approaches. Eur J Cardiothorac Surg. 2010;37:170-85.

12. Fedoruk LM, Tribble CG, Kern JA, Peeler BB, Kron IL. Predicting operative mortality after surgery for ischemic cardiomyopathy. Ann Thorac Surg. 2007; 83:2029-35.

13. Ngaage DL, Daly RC, Rosales G, Sundt TM 3rd, Dearani JA, Mullany CJ, et al. Mitral regurgitation surgery in heart failure due to ischemic cardiomyopathy: a 24-year experience. J Heart Valve Dis. 2008;17:251-60.

14. Flameng W, Szecsi J, Sergeant P, Daenen W, Herijgers P, Scheys I. Combined valve and coronary artery bypass surgery: early and late results. Eur J Cardiothorac Surg. 1994;8:410-9.

15. Paparella D, Malvindi PG, Romito R, Iacoviello M, Visicchio G, De Serio F, et al. $\mathrm{BNP}$ in mitral valve restrictive annuloplasty for ischemic mitral regurgitation. Int J Cardiol. 2009;137:57-60.

16. Dahlberg PS, Orszulak TA, Mullany CJ, Daly RC, Enriquez-Sarano M, Schaff HV. Late outcome of mitral valve surgery for patients with coronary artery disease. Ann Thorac Surg. 2003;76:1539-48; discussion 1547-8.

17. Gelsomino S, Lorusso R, De Cicco G, Capecchi I, Rostagno C, Caciolli S, et al. Five-year echocardiographic results of combined undersized mitral ring annuloplasty and coronary artery bypass grafting for chronic ischaemic mitral regurgitation. Eur Heart J. 2008;29:231-40.

18. van Garsse L, Gelsomino S, Luca F, Lorusso R, Rao CM, Stefàno $\mathrm{P}$, et al. Importance of anterior leaflet tethering in predicting recurrence of ischemic mitral regurgitation after restrictive annuloplasty. J Thorac Cardiovasc Surg. 2012;143:S54-9.

19. McGee EC, Gillinov AM, Blackstone EH, Rajeswaran J, Cohen G, Najam F, et al. Recurrent mitral regurgitation after annuloplasty for functional ischemic mitral regurgitation. J Thorac Cardiovasc Surg. 2004;128:916-24.

20. Parker JA, Kennes LN, Ruckert J, Dahm M, Vahl CF. Outcome after mitral valve operations with depressed left ventricular function. Asian Cardiovasc Thorac Ann. 2012;20:292-8.

21. Bishay ES, McCarthy PM, Cosgrove DM, Hoercher KJ, Smedira NG, Mukherjee D, et al. Mitral valve surgery in patients with severe left ventricular dysfunction. Eur J Cardiothorac Surg. 2000;17:213-21. 